\title{
Correlação entre as características do perfil e desenvolvimento sensório-motor de crianças com síndromes genéticas
}

The correlation between characteristics of the profile and sensory-motor development of children with genetic syndromes

\author{
Correlación entre las características del perfil y desarrollo sensorio-motriz de niños con síndromes genéticos
}

Ana Paula Vasconcelos Alves ${ }^{1}$, Cibelle Kayenne Martins Roberto Formiga², Fabiana Pavan Viana ${ }^{3}$

\section{RESUMO}

Este trabalho trata-se de estudo transversal cujo objetivo foi verificar correlação entre as características do perfil e desenvolvimento sensório-motor de crianças com síndromes genéticas. Para tal, realizou-se pesquisa entre agosto e dezembro de 2009, com treze portadores de síndromes genéticas que frequentam a Associação Pestalozzi em Goiânia. Os instrumentos utilizados foram: Inventário Portage (IP), Ficha de Avaliação Neurológica Infantil e Gross Motor Function Classification System (GMFCS). Na análise estatística realizou-se teste de correlação de Spearman. Observou-se que quanto maior a idade da criança, maior o grau de incapacidade e nível de classificação funcional (GMFCS) e menor o percentual de desempenho nas áreas do IP. Quanto maior o peso da criança ao nascer, mais precoce a aquisição de habilidades motoras, tais como sentar, engatinhar e ficar em pé. Assim, verifica-se a importância de abordagem global no atendimento à criança, que deve incluir tanto aspectos neurofuncionais como estimulação do desenvolvimento sensório-motor.

Descritores: Desenvolvimento Infantil; Fisioterapia; Doenças Genéticas Inatas.

\section{ABSTRACT}

This cross-sectional study was performed with the objective to verify the correlation between the characteristics of the profile and sensory-motor development of children with genetic syndromes. A research was performed between August and December of 2009, with 13 patients with genetic syndromes undergoing treatment at Associação Pestalozzi de Goiânia (GO). The instruments used were: Portage Inventory (PI), Children's Neurology Assessment Form, and Gross Motor Function Classification System (GMFCS). Statistical analysis was performed using Spearman's correlation test. It was observed that older children had a higher degree of incapacity, higher level of functional classification on the GMFCS, and a poorer performance rate on PI areas. The higher the birthweight, the earlier the child's need to acquire motor skills, such as sitting, crawling and standing. Therefore, it is important to assess and treat children using a global approach, which should include neurofunctional aspects as well as stimulation for their sensory-motor development.

Descriptors: Child Development; Physical Therapy Specialty; Genetic Diseases, Inborn.

\section{RESUMEN}

Estudio transversal que objetivó verificar la correlación entre características del perfil y desarrollo sensorio-motriz de niños con síndromes genéticos. Investigación realizada entre agosto/diciembre 2009, con 13 portadores de síndromes genéticos, pacientes de la Asociación Pestalozzi, Goiânia-GO. Se utilizaron los instrumentos: Inventario Portage (IP), Ficha de Evaluación Neurológica Infantil y Gross Motor Function Classification System (GMFCS). En análisis estadístico se realizó test de correlación de Spearman. Se observó que cuanto mayor es la edad del niño, mayor es el grado de incapacidad, así como es mayor el nivel de clasificación funcional por GMFCS y menor el porcentaje de desempeño en las áreas del IP. A mayor peso de los niños al nacer, más precoz es la adquisición de habilidades motoras, como sentarse, gatear y pararse. En consecuencia, se verifica la importancia de un abordaje global de atención al niño, que debe incluir tanto aspectos neurofuncionales como la estimulación del desarrollo sensorio-motriz.

Descriptores: Desarrollo Infantil; Fisioterapia; Enfermedades Genéticas Congénitas.

\footnotetext{
${ }^{1}$ Fisioterapeuta. Goiânia, GO, Brasil. E-mail: paulinha57@uol.com.br.

${ }^{2}$ Fisioterapeuta, Doutora em Ciências Médicas. Professora Titular, Universidade Estadual de Goiás. Goiânia, GO, Brasil. E-mail: cibellekayenne@gmail.com.

${ }^{3}$ Fisioterapeuta, Doutora em Ciências Fisiológicas. Professora Adjunto I, Pontifícia Universidade Católica de Goiás. Goiânia, GO, Brasil. E-mail: pmfviana@gmail.com.
} 


\section{INTRODUÇÃO}

Define-se como desenvolvimento infantil um processo que se inicia desde a vida intrauterina, sendo este influenciado por fatores como a maturação neurológica, o crescimento físico e a construção de habilidades relacionadas ao comportamento e as esferas cognitivas, afetivas e sociais da criança(1). Dessa forma, os primeiros anos de vida são de importância fundamental, pois se caracterizam por aquisições importantes e pela grande plasticidade neuronal que propicia avanços nas áreas motoras, cognitivas e de linguagem ${ }^{(2)}$.

A maturação do sistema nervoso central (SNC) e os inputs sensoriais são fatores biológicos que favorecem o desenvolvimento neuropsicomotor. Os inputs sensoriais estão relacionados às funções receptivas, tais como a capacidade para selecionar, adquirir, classificar e integrar as informações, sendo organizados através da experimentação de sensações que serão oferecidas pelo ambiente e proporcionarão respostas adaptativas, que se aprimorarão com o decorrer do tempo(3).

A susceptibilidade para o atraso no desenvolvimento neuropsicomotor é determinada por fatores de risco que agem na primeira infância ${ }^{(1)}$, sendo os domínios sensóriomotor, cognitivo e sócio-emocionais fortemente influenciados por fatores biológicos, como por exemplo, a idade gestacional e o peso do bebê ao nascimento, bem como por fatores socioambientais e genéticos(4).

Dentre as condições de risco genéticas, as desordens médicas definidas destacam-se, pois a ocorrência de síndromes genéticas interfere no desenvolvimento infantil, podendo desencadear atrasos como a incapacidade na execução de atividades motoras. No que tange às mesmas, os distúrbios cromossômicos assumem papel de destaque, uma vez que afetam cerca de sete para cada 1000 nascidos vivos, e são responsáveis por alterações reprodutivas, malformações e retardo mental(5). Além do mais, a ocorrência mais frequente dentre todas as síndromes genéticas que geram má formação nos seres humanos é a Síndrome de Down ${ }^{(6)}$.

Crianças com alterações do desenvolvimento necessitam de assistência que englobe tanto a sua doença de base quanto às limitações impostas(7). A atuação profissional com essas crianças deve ser feita por uma equipe multidisciplinar formada por fisioterapeuta, terapeuta ocupacional, fonoaudiólogo, enfermeiros e equipe médica. Todos devem se voltar para a manutenção da saúde e estimular o potencial de desenvolvimento global da criança, fazendo com que a criança com atraso se torne apta a responder as suas necessidades, bem como as do seu meio ${ }^{(8)}$.

Por conseguinte, torna-se importante aprofundar o conhecimento a respeito das características do perfil de crianças com síndromes genéticas e o impacto destas no desenvolvimento sensório-motor. A pesquisa sobre tal temática facilitará a elaboração de programas que priorizem o desenvolvimento saudável e estratégias de tratamento de reabilitação dos portadores de síndrome genética.

Neste sentido, os objetivos do estudo foram verificar as associações entre as características do perfil com o desenvolvimento sensório-motor de crianças com síndromes genéticas.

\section{MÉTODOS}

Foi realizado um estudo transversal com uma amostra de conveniência de 13 crianças portadoras de síndromes genéticas que frequentam o setor de Fisioterapia da Associação Pestalozzi de Goiânia (Unidade Renascer) de agosto a dezembro de 2009. Os participantes considerados elegíveis para o estudo foram crianças com diagnóstico clínico de síndromes genéticas, independente do grau de deficiência mental, de ambos os sexos, com idade de um a 12 anos que realizam tratamento fisioterapêutico regular na instituição, cujos pais e/ou responsáveis aceitaram participar da pesquisa.

Os critérios de exclusão foram presença de malformações congênitas de membros, crianças clinicamente instáveis no momento das avaliações (exemplos: problemas respiratórios, infecções). A amostra de estudo avaliou todas as crianças que frequentavam a instituição no período matutino.

Os instrumentos utilizados na pesquisa foram o Inventário Portage (IP), a Ficha de Avaliação Neurológica Infantil e o Gross Motor Function Classification System (GMFCS).

O IP foi elaborado e introduzido experimentalmente em Portage (Wisconsin, EUA) em 1972. No Brasil, foi adaptado por duas psicólogas brasileiras, que traduziram e operacionalizaram seus itens, propondo-lhes definições, critérios, especificando as condições de avaliação e descrevendo o material a ser utilizado para estudos e aplicação clínica ${ }^{(9)}$. O instrumento é 
constituído por 580 itens no total, que visam avaliar o desenvolvimento neuropsicomotor de crianças de zero a seis anos de idade, nas seguintes áreas: socialização, cognição, linguagem, autocuidados e desenvolvimento motor. Além disso, o IP possui uma área de estimulação infantil usada para avaliar bebês de zero a quatro meses. O IP possui itens que devem ser avaliados diretamente com a criança e outros itens que podem ser registrados por meio de entrevista com os cuidadores ${ }^{(9)}$. Diversos trabalhos científicos descrevem a aplicação do IP como medida de avaliação do desempenho neuro-sensóriomotor de bebês e crianças com atrasos e/ou anormalidades no desenvolvimento global(10-11).

Apesar de ser destinado para a avaliação do desenvolvimento até seis anos de idade, o IP foi utilizado no presente estudo em virtude do atraso e grau de incapacidade funcional apresentado pelas crianças. Cada criança foi avaliada de acordo com a faixa etária em que se encontrava. Se a criança apresentasse dificuldade em realizar até cinco itens na mesma sequência, a faixa etária anterior era utilizada como referência. A pontuação do IP foi calculada conforme o número de acertos obtidos pela criança dividido pelo número de itens considerados de acordo com a idade. O resultado obtido foi multiplicado por 100 para a obtenção da porcentagem de desempenho.

A Ficha de Avaliação Neurológica Infantil é uma ficha padronizada para avaliação dos pacientes que realizam tratamento no Setor de Fisioterapia da instituição. A ficha contém dados sobre a anamnese, exame físico e neurológico. Foram avaliadas questões como a história da moléstia atual, história familiar, exame físico, avaliação do crescimento e desenvolvimento, condições do parto e da gravidez.

O GMFCS é um sistema de classificação funcional desenvolvido no Canadá, em um centro de reabilitação para a infância. O instrumento proporciona uma descrição válida e segura da função motora de crianças, bem como habilita o acoplamento dos dados a informação sobre prognóstico motor total e as implicações para dimensionar sua participação na sociedade ${ }^{(12)}$. É constituído por cinco níveis, sendo uma escala ordinal, em que para cada nível são fornecidas descrições para crianças em diferentes faixas etárias (menores de dois anos, de dois a quatro anos, de quatro a seis anos e de seis a 12 anos). O sistema de classificação proposto tem sido usado internacionalmente em vários estudos com crianças com disfunções motoras. As distinções entre os níveis de função motora são baseadas nas limitações funcionais e de aparelhos auxiliares de locomoção(13).

Para a realização do estudo, o procedimento inicial foi esclarecer os pais das crianças sobre a pesquisa e obter autorização da participação por meio da assinatura do Termo de Consentimento Livre e Esclarecido. As fichas de Avaliação Neurológica Infantil das crianças selecionadas foram examinadas e os dados da anamnese e exame neurofuncional foram registrados. Posteriormente, cada criança foi avaliada individualmente na sala de Fisioterapia da instituição, classificada de acordo com os critérios do GMFCS, e seus comportamentos foram avaliados por meio das áreas do desenvolvimento do IP. A avaliação durou uma média de 40 minutos.

Durante a avaliação as crianças vestiam roupas confortáveis que permitiam uma total liberdade dos movimentos. Todas elas foram colocadas sobre o colchonete para a avaliação fisioterapêutica e foram utilizados brinquedos e materiais pedagógicos para estimular as mudanças de decúbito e habilidades específicas nas áreas de estimulação infantil, socialização, desenvolvimento motor, cognitivo e linguagem. Todas as avaliações foram realizadas por uma equipe treinada de alunos de iniciação científica do curso de fisioterapia e supervisionadas pela autora (CKMRF) durante a aplicação dos instrumentos. Ressalta-se que a coleta de dados do presente estudo não interferiu na rotina de atendimentos semanais estabelecida pela instituição.

A análise estatística dos dados foi feita pelo programa Statistical Package for Social Science (SPSS) versão 10.0. Na análise da correlação entre as variáveis foi utilizado o Teste de Correlação de Spearman para verificar a associação entre o perfil e o desenvolvimento sensório-motor. Foi adotado um nível de significância de $5 \%$.

Foram incluídas no teste de correlação as seguintes variáveis do perfil das crianças: idade das crianças, nível funcional do GMFCS, grau de incapacidade (leve, moderado e grave), idade em que os pais notaram o atraso da criança, peso ao nascimento, áreas do IP (estimulação infantil, desenvolvimento motor, cognição, linguagem, socialização e autocuidados). 
As variáveis do desenvolvimento sensório-motor incluídas foram: áreas do IP, controle postural sentado sem apoio, controle postural sentado com apoio, idade que engatinhou, idade que rolou, idade que ficou em pé com apoio, idade que iniciou a reabilitação, reação de proteção para frente, proteção para lados, proteção para trás, sentado com apoio, sentado sem apoio, sustento cefálico, tônus de membros superiores (MMSS), tônus de membros inferiores (MMII), transferência de supino para sentado, prono para sentado, sentado para gato, gato para sentado, gato para ajoelhado, ajoelhado para gato e o uso de cadeira de rodas.

Este trabalho foi aprovado pelo Comitê de Ética e Pesquisa da Pontifícia Universidade Católica de Goiás (PUC-Goiás) (protocolo número 0769.0.000.168-07).

\section{RESULTADOS}

A idade média das crianças com síndromes genéticas foi de quatro anos ( $D P=2,9$ anos), sendo que $46 \%$ ( $n=6)$ eram do sexo feminino e $54 \%(n=7)$ do sexo masculino. No que tange a prevalência das síndromes genéticas, verificou-se que $46 \%$ ( $n=6$ ) possuíam síndrome de Down e a porcentagem de $8 \%(n=1)$ foi encontrada para as síndromes de Angelman, do Cromossomo 1, do Cromossomo 11, de Lenox Gastaud, de Cohen, de West e de Cri Du Chat.

Quanto aos dados de nascimento das crianças, 85\% ( $n=11)$ nasceram de parto cesárea, média de peso ao nascer de 2,592 gramas (DP=1,010g), média do Apgar no primeiro minuto de $6,8(D P=1,2)$ e no quinto minuto de $8,4(\mathrm{DP}=0,6)$.

Os resultados da avaliação do desenvolvimento sensório-motor pelo IP encontram-se na Tabela 1.

Tabela 1: Pontuação das crianças na avaliação do desenvolvimento sensório-motor segundo o IP. Goiânia, GO, 2009.

\begin{tabular}{ccccc}
\hline Áreas Avaliadas (IP) & Média de acertos (\%) & DP & Mínimo & Máximo \\
\hline Estimulação infantil & 81,5 & 18,6 & 38,0 & 100,0 \\
Desenvolvimento motor & 39,1 & 26,7 & 1,0 & 78,0 \\
Socialização & 38,4 & 32,3 & 2,4 & 95,0 \\
Autocuidados & 27,0 & 29,2 & 2,0 & 92,0 \\
Cognição & 22,3 & 27,0 & 1,0 & 27,0 \\
Linguagem & 8,9 & 19,1 & 1,0 & 70,0 \\
Pontuação total & 36,2 & 22,5 & 9,5 & 82,0 \\
\hline
\end{tabular}

IP=Inventário Portage; DP=desvio-padrão.

Observa-se que as crianças apresentaram média de desempenho (acertos) abaixo do esperado (80 a 100\%) para a faixa etária nas áreas avaliadas, exceto na área de estimulação infantil $(81,5 \%)$ e na pontuação total.
Os resultados da classificação das crianças de acordo com a avaliação do GMFCS encontram-se na Tabela 2.

Tabela 2: Classificação das crianças portadoras de síndromes genéticas com relação ao nível funcional avaliado pelo GMFCS. Goiânia, GO, 2009.

\begin{tabular}{cccc}
\hline Classificação funcional (GMFCS) & f & $\%$ \\
\hline Nível I & 2 & 15 & 15 \\
Nível II & 2 & 15 & 2 \\
Nível III & 50 & 15 \\
\hline
\end{tabular}

f=frequência absoluta; \%=porcentagem.

A maioria das crianças apresentou maior grau de limitação funcional (níveis III, IV e V) pelo GMFCS.

As correlações encontradas entre a idade das crianças, classificação do GMFCS, grau de incapacidade e idade que os pais notaram o atraso, com os dados da avaliação neurofuncional e IP estão apresentadas na Tabela 3. 
Tabela 3: Correlação entre idade das crianças, GMFCS, grau de incapacidade e início da reabilitação com os dados da avaliação neurofuncional e IP. Goiânia, GO, 2009.

\begin{tabular}{|c|c|c|c|}
\hline Perfil das crianças & Avaliação neurofuncional & $\mathbf{r}$ & $\mathbf{p}$ \\
\hline & Grau de incapacidade & 0,78 & 0,002 \\
\hline \multirow[t]{5}{*}{ Idade das crianças (anos) } & Idade que controlou a cabeça & 0,56 & 0,045 \\
\hline & Área de cognição do IP & $-0,68$ & 0,011 \\
\hline & Grau de incapacidade & 0,55 & 0,049 \\
\hline & Tônus cervical e tronco & $-0,69$ & 0,009 \\
\hline & Sustento cefálico & 0,66 & 0,015 \\
\hline \multirow[t]{5}{*}{ Nível funcional do GMFCS } & Desenvolvimento motor do IP & $-0,83$ & 0,001 \\
\hline & Autocuidados do IP & $-0,71$ & 0,007 \\
\hline & Cognição do IP & $-0,56$ & 0,046 \\
\hline & Pontuação Total do IP & $-0,67$ & 0,012 \\
\hline & Idade que rolou & 0,70 & 0,008 \\
\hline \multirow[t]{4}{*}{ Grau de incapacidade } & Área de autocuidados do IP & $-0,70$ & 0,008 \\
\hline & Área de cognição do IP & $-0,70$ & 0,005 \\
\hline & Idade que iniciou reabilitação & 0,72 & 0,005 \\
\hline & Transferência de supino para sentado & $-0,68$ & 0,009 \\
\hline \multirow[t]{3}{*}{ Idade que os pais notaram atraso (meses) } & Transferência de prono para sentado & $-0,68$ & 0,009 \\
\hline & Transferência de ajoelhado para semi-ajoelhado & $-0,66$ & 0,013 \\
\hline & Transferência de semi-ajoelhado para ajoelhado & $-0,66$ & 0,013 \\
\hline
\end{tabular}

$r=$ coeficiente de Spearman, $p<0,05$.

A relação entre a idade das crianças e os dados da avaliação neurofuncional demonstrou que quanto maior a idade da criança, maior o grau de incapacidade e maior o tempo para a aquisição do controle de cabeça. De acordo com o nível funcional do GMFCS, as crianças com maior nível de classificação apresentaram maior grau de incapacidade, bem como obtiveram menor percentual nas áreas de desenvolvimento motor, autocuidados, cognição e pontuação total do IP. Verificou-se que quanto mais tarde foi notado o atraso no desenvolvimento, maior a idade de início do tratamento, assim como a incapacidade para a realização de transferências posturais.

Os resultados da correlação entre o peso da criança ao nascer e a alteração do tônus cervical e tronco com os dados da avaliação neurofuncional estão dispostos na Tabela 4.

Tabela 4: Correlação entre peso da criança e tônus cervical e tronco com os dados da avaliação neurofuncional. Goiânia, GO, 2009.

\begin{tabular}{|c|c|c|c|}
\hline Perfil das crianças & Avaliação neurofuncional & $\mathbf{r}$ & $\mathbf{p}$ \\
\hline \multirow{4}{*}{ Peso da criança ao nascer (g) } & Idade que sentou & $-0,65$ & 0,014 \\
\hline & Idade que engatinhou & $-0,62$ & 0,022 \\
\hline & Idade que ficou em pé & $-0,57$ & 0,039 \\
\hline & Transferência de supino para sentado & 0,64 & 0,017 \\
\hline \multirow{9}{*}{ Tônus cervical e tronco } & Transferência de prono para sentado & 0,64 & 0,017 \\
\hline & Tônus de MMSS & 0,62 & 0,025 \\
\hline & Tônus de MMII & 0,62 & 0,025 \\
\hline & Proteção para frente & $-0,84$ & 0,001 \\
\hline & Proteção para os lados & $-0,84$ & 0,001 \\
\hline & Proteção para trás & $-0,60$ & 0,03 \\
\hline & Sentado com apoio & $-0,82$ & 0,001 \\
\hline & Sentado sem apoio & $-0,60$ & 0,03 \\
\hline & Sustento cefálico & $-0,82$ & 0,001 \\
\hline
\end{tabular}

$r=$ coeficiente de Spearman, $p<0,05$.

Os resultados mostraram uma correlação significativa entre o peso da criança ao nascer e as aquisições motoras, sendo que quanto maior o peso de nascimento mais precoce a aquisição dos movimentos de sentar, engatinhar, ficar em pé e possuem maior capacidade em realizar transferências posturais. Além 
do mais, verificou-se que as crianças com alteração do tônus cervical e tronco também apresentavam alteração do tônus de MMSS e MMII, bem como maior atraso na aquisição de habilidades motoras, tais como o sustento cefálico, o controle postural sentado com e sem apoio, e presença de reações de proteção.

A correlação entre as áreas do IP e os dados da avaliação neurofuncional foram detalhados na Tabela 5.

Tabela 5: Correlação das áreas do IP com dados da avaliação neurofuncional. Goiânia, GO, 2009.

\begin{tabular}{|c|c|c|c|}
\hline Áreas do IP & Avaliação Neurofuncional & $\mathbf{r}$ & $\mathbf{p}$ \\
\hline \multirow{10}{*}{ Estimulação infantil } & Desenvolvimento motor (IP) & 0,6 & 0,029 \\
\hline & Linguagem (IP) & 0,8 & 0,001 \\
\hline & Socialização (IP) & 0,87 & 0,001 \\
\hline & Pontuação final (IP) & 0,74 & 0,004 \\
\hline & Controle postural sentado sem apoio & $-0,57$ & 0,041 \\
\hline & Transferência de supino para sentado & $-0,58$ & 0,037 \\
\hline & Transferência de prono para sentado & $-0,58$ & 0,037 \\
\hline & Socialização (IP) & 0,77 & 0,002 \\
\hline & Autocuidados (IP) & 0,82 & 0,001 \\
\hline & Cognição (IP) & 0,78 & 0,002 \\
\hline \multirow[t]{6}{*}{ Desenvolvimento motor } & Pontuação total (IP) & 0,87 & 0 \\
\hline & Controle postural sentado com apoio & $-0,64$ & 0,019 \\
\hline & Controle postural sentado sem apoio & $-0,74$ & 0,004 \\
\hline & Transferência supino para sentado & $-0,76$ & 0,003 \\
\hline & Transferência de prono para sentado & 0,76 & 0,003 \\
\hline & Transferência de sentado para gato & $-0,82$ & 0,001 \\
\hline \multirow{4}{*}{ Cognição } & Transferência de gato para sentado & $-0,82$ & 0,001 \\
\hline & Transferência de gato para ajoelhado & $-0,63$ & 0,02 \\
\hline & Transferência de ajoelhado para gato & $-0,63$ & 0,02 \\
\hline & Pontuação total (IP) & 0,87 & 0,001 \\
\hline \multirow{3}{*}{ Linguagem } & Socialização (IP) & 0,8 & 0,001 \\
\hline & Pontuação total (IP) & 0,71 & 0,007 \\
\hline & Autocuidados (IP) & 0,67 & 0,012 \\
\hline \multirow[t]{3}{*}{ Socialização } & Cognição (IP) & 0,76 & 0,002 \\
\hline & Pontuação total (IP) & 0,92 & 0,001 \\
\hline & Cognição (IP) & 0,93 & 0,001 \\
\hline \multirow{3}{*}{ Autocuidados } & Pontuação total (IP) & 0,83 & 0,001 \\
\hline & Transferência de gato para ajoelhado & $-0,59$ & 0,033 \\
\hline & Transferência ajoelhada para gato & $-0,59$ & 0,033 \\
\hline
\end{tabular}

$r=$ coeficiente de Spearman; $p<0,05$.

Os resultados demonstraram que houve associação entre as áreas do IP, uma vez que as crianças que obtiveram maior pontuação em uma determinada área, também as alcançaram nas demais. Além disso, uma maior pontuação nas áreas de estimulação infantil, desenvolvimento motor e autocuidados, mostrou associação com menor idade na aquisição de habilidade na realização das transferências posturais.

\section{DISCUSSÃO}

A idade média das crianças com síndromes genéticas foi de quatro anos, sendo que a maioria era portadora de Síndrome de Down e do sexo masculino. Observa-se que dentre as doenças genéticas, a Síndrome de Down possui maior incidência, com proporção de um para cada
$600 / 800$ nascidos vivos e estima-se que existam cerca de 300.000 pessoas no Brasil com esta anomalia genética ${ }^{(14)}$.

As crianças apresentaram desenvolvimento sensório-motor abaixo do esperado para a faixa etária em todas as áreas avaliadas, exceto para a área de estimulação infantil. Estes resultados podem ser confirmados pelo fato de todas as crianças apresentarem maior ou menor grau de deficiência mental, o que prejudica a realização de atividades do cotidiano, especialmente no que se refere as capacidades cognitivas, de linguagem e autocuidados ${ }^{(11,15)}$.

Os resultados obtidos neste trabalho demonstraram que crianças quanto maior o nível de classificação das 
crianças no GMFCS, maior o grau de incapacidade apresentado pelas crianças, bem como menor o percentual nas áreas de desenvolvimento motor, autocuidados, cognição e pontuação total do IP. Dessa forma, verifica-se que os atrasos no desenvolvimento também podem estar relacionados a limitações nas habilidades funcionais. São exemplos de atividades funcionais: o autocuidado (alimentação e banho independentes), as tarefas de função social (ir à escola e interagir com outras crianças) e as atividades de mobilidade como levantar da cama e ir ao banheiro com independência ${ }^{(16)}$.

A análise dos dados demonstrou que há uma relação negativa do comprometimento motor grave no desempenho das atividades do cotidiano com a independência funcional das crianças, uma vez que aspectos intrínsecos e extrínsecos limitam suas possibilidades funcionais, dificultando o desempenho de atividades do cotidiano. Por conseguinte, assevera-se que o ambiente é aspecto fundamental para o desenvolvimento, pois pode moldar aspectos do comportamento motor e agir como facilitador, pois possibilita a exploração e interação com o meio(17).

Outro resultado relevante encontrado demonstra que quanto mais tarde é notado o atraso no desenvolvimento, maior é a idade de início do tratamento, assim como a incapacidade para a realização de transferências posturais. Em um estudo de revisão verificou-se que diversos fatores podem interferir no curso normal do desenvolvimento motor. Contudo, a intervenção adequada pode ser benéfica para prevenir, minimizar ou até mesmo reverter o atraso no desenvolvimento ${ }^{(18)}$.

A análise dos resultados mostrou uma correlação significativa entre o peso da criança ao nascer e as aquisições motoras, sendo quanto maior o peso das crianças ao nascer, menor o tempo para aquisição de habilidades motoras como sentar, engatinhar, ficar em pé, e maior a capacidade em realizar transferências posturais. O nascimento com baixo peso e, especialmente, com muito baixo peso (inferior a $1.500 \mathrm{~g}$ ) pode comprometer áreas do desenvolvimento, desencadeando problemas de linguagem, de aprendizagem e de coordenação viso-motora. A avaliação do desenvolvimento neuropsicomotor no primeiro ano de vida, principalmente nas situações de risco biológico, é fundamental para lactentes em situação de vulnerabilidade ${ }^{(19)}$.

No presente estudo, houve correlação significativa entre a área de linguagem e socialização do IP. O desenvolvimento da linguagem verbal depende da interação entre as capacidades biológicas e estimulação ambiental, bem como a sua evolução está associada à progressão do desenvolvimento neuropsicomotor ${ }^{(20)}$. Os resultados também demonstraram que quanto maior a pontuação das crianças em uma determinada área do IP, maior a pontuação obtida nas demais áreas do IP. Da mesma forma, quanto maior a pontuação das crianças nas áreas de estimulação infantil, desenvolvimento motor e autocuidados, menor o tempo das crianças para aquisição de habilidades na realização das transferências posturais.

Em estudo comparativo realizado com 20 crianças portadoras de Síndrome de Down e 20 com desenvolvimento normal verificou que indivíduos com Síndrome de Down apresentam desempenho significativamente inferior nas seguintes áreas de habilidades funcionais, autocuidado, função social e mobilidade ${ }^{(15)}$. O referido estudo apresenta semelhança com os resultados encontrados, tendo em vista que as crianças apresentaram desempenho considerado atrasado nos aspectos avaliados.

Por conseguinte, verifica-se que os domínios do IP estão relacionados entre si e interferem conjuntamente no desenvolvimento das crianças, apresentando uma relação com o desempenho das atividades do cotidiano e na independência funcional da criança. Tal fato reforça a necessidade de se considerar a variabilidade desses pacientes e suas particularidades na prática clínica. O desenvolvimento motor das crianças passa por vários estágios, sendo indispensável propiciar à criança a oportunidade de se desenvolver da melhor forma possível, por meio de atividades que permitam a organização do comportamento sensório-motor, bem como a formação social(6).

A partir dos resultados obtidos conclui-se que houve correlação entre as características do perfil com o desenvolvimento sensório-motor das crianças com síndromes genéticas participantes do estudo, principalmente no que se refere às atividades funcionais e aspectos da função neurológica. Pode-se apontar como limitações do estudo o pequeno tamanho amostral e a participação de crianças com diferentes diagnósticos. 
Neste sentido, os achados se limitam às características da amostra estudada, não podendo ser generalizado para esta população de crianças. Apesar dessas limitações, os resultados obtidos podem contribuir para

\section{REFERÊNCIAS}

1. Saccani R, Brisola E, Giordini AP, Bach S, Resende TL, Almeida CS. Avaliação do desenvolvimento neuropsicomotor em crianças de um bairro da periferia de Porto Alegre. Scientia Medica [Internet]. 2007 [cited 2012 jun 30];17(3):130-7. Available from:

http://revistaseletronicas.pucrs.br/ojs/index.php/scientiamedica/art icle/viewFile/1657/2145.

2. Maria-Mengel MRS, Linhares MBM. Fatores de risco para problemas de desenvolvimento infantil. Rev Lat Am Enfermagem [Internet]. 2007 [cited 2012 jun 30];15(spe):837-42. Available from: http://dx.doi.org/10.1590/S0104-11692007000700019.

3. Paiva GS, Lima AC, Lima Mde C, Eickmann SH. The effect of poverty on developmental screening scores among infants. Sao Paulo Med J [Internet]. 2010 [cited 2012 jun 30];128(5):276-83. Available from: http://dx.doi.org/10.1590/S151631802010000500007.

4. Santos DCC, Tolocka RE, Carvalho J, Heringer LRC, Almeida CM, Miquelote AF. Desempenho motor e sua associação com fatores neonatais, familiares e de exposição à creche em crianças até três anos de idade. Rev. bras. fisioter. [Internet]. 2009 [cited 2012 jun 30];13(2):173-9. Available from: http://dx.doi.org/10.1590/S141335552009005000025 .

5. Nakata NMK. Síndromes genéticas e ambientais em distúrbios da audição [thesis ]. Bauru: Universidade de São Paulo/ USP; 2006. 6. Florentino Neto J, Fernandes Filho J, De Pontes LM. Impacto de 12 semanas de treinamento de força sobre a composição corporal de portadores da síndrome de Down. Revista da AMRIGS 2009;53(1):11-5.

7. Miranda LP, Resegue R, Figueiras ACM. A criança e o adolescente com problemas do desenvolvimento no ambulatório de pediatria. J Pediatr (Rio J) [Internet]. 2003 [cited 2012 jun 30];79 Suppl 1:S33-42. Available from: http://dx.doi.org/10.1590/S002175572003000700005.

8. Copetti F, Mota CB, Graup S, Menezes KM, Venturini EB. Comportamento angular do andar de crianças com síndrome de Down após intervenção com equoterapia. Rev. bras. fisioter. [Internet]. 2007 [cited 2012 jun 30];11(6):503-7. Available from: http://dx.doi.org/10.1590/S1413-35552007000600013.

9. Williams LCA, Aiello ALR. O Inventário Portage Operacionalizado: Intervenção com famílias. São Paulo: MEMNON/ FAPESP; 2001.

10. Formiga CKMR, Pedrazzani ES, Silva FPs, Lima CD. Eficácia de um programa de intervenção precoce com bebês pré-termo e suas famílias. Paidéia (Ribeirão Preto) [Internet]. 2004 [cited 2012 jun 30];14(29): 301-11. Available from: http://dx.doi.org/10.1590/S0103863X2004000300006.

11. Alves APV, Formiga CKMR, Viana FP. Perfil e desenvolvimento de crianças com síndromes genéticas em tratamento multidisciplinar. Rev Neurocienc [Internet]. 2011 [cited 2012 jun 30];19(2):284-93. Available from:

http://www.revistaneurociencias.com.br/edicoes/2011/RN1902/rela to $\% 20$ de $\% 20$ caso $\% 2019 \% 2002 / 467 \% 20$ relato $\% 20$ de $\% 20$ caso.pdf. 12. Palisano R, Rosenbaum P, Walter S, Russell D, Wood E, Galuppi B. Development and reliability of a system to classify gross motor function in children with cerebral palsy. Dev Med Child Neurol. 1997;39(4):214-23.

13. Vasconcelos RLM, Moura TL, Campos TF, Lindquist, ARR, Guerra RO. Avaliação do desempenho funcional de crianças com paralisia cerebral de acordo com níveis de comprometimento motor. Rev. bras. fisioter. [Internet]. 2009 [cited 2012 jun 30];13(5):390-7. Available from: http://dx.doi.org/10.1590/S141335552009005000051 . 14. Silva MFMC, Kleinhans ACS. Processos cognitivos e plasticidade cerebral na Síndrome de Down. Rev. bras. educ. espec. [Internet]. o conhecimento dos profissionais envolvidos no atendimento à saúde e reabilitação neurológica dessas crianças, tais como enfermeiros, fisioterapeutas, terapeutas ocupacionais e fonoaudiólogos.

2006 [cited 2012 jun 30];12(1):123-38. Available from: http://dx.doi.org/10.1590/S1413-65382006000100009. 15. Pazin AC, Martins MRI. Desempenho funcional de crianças com Síndrome de Down e a qualidade de vida de seus cuidadores. Rev Neurocienc [Internet]. 2007 [cited 2012 jun 30];15(4):297-303. Available from:

http://www.revistaneurociencias.com.br/edicoes/2007/RN\%2015\%2 004/Pages\%20from\%20RN\%2015\%2004-8.pdf.

16. Malta J, Endriss D, Rached S, Moura T, Ventura L. Desempenho funcional de crianças com deficiência visual, atendidas no Departamento de Estimulação Visual da Fundação Altino Ventura. Arq Bras Oftalmol [cited 2012 jun 30]. 2006 [cited 2012 jun 30];69(4):571-4. Available from: http://dx.doi.org/10.1590/S000427492006000400021.

17. Silva PL, Santos DCC, Gonçalves VMG. Influência de práticas maternas no desenvolvimento motor de lactentes do $6^{\circ}$ ao $12^{\circ}$ meses de vida. Rev. bras. fisioter. [Internet]. 2006 [cited 2012 jun 30];10(2):225-31. Available from: http://dx.doi.org/10.1590/S141335552006000200014.

18. Willrich A, Azevedo CCF, Fernandes JO. Desenvolvimento motor na infância: influência dos fatores de risco e programas de intervenção. Rev Neurocienc [Internet]. 2009 [cited 2012 jun 30];17(1):51-6. Available from:

19. Nascimento R, Piassão C. Avaliação e estimulação do desenvolvimento neuropsicomotor em lactentes institucionalizados. Rev Neurocienc [Internet]. 2010 [cited 2012 jun 30];18(1):469-78. Available from:

http://www.revistaneurociencias.com.br/edicoes/2009/RN\%202009 $\% 201 / 226 \% 20 . p d f$

20. Schirmer CR, Fontoura DR, Nunes ML. Distúrbios da aquisição da linguagem e da aprendizagem. J Pediatr (Rio J) [Internet]. 2004 [cited 2012 jun 30];80(2):95-103. Availlable from: http://dx.doi.org/10.1590/S0021-75572004000300012.

Artigo recebido em 11/08/2011. Aprovado para publicação em 16/04/2012. Artigo publicado em 30/06/2012. 